\title{
El concepto de autoría corporativa de Panizzi a Cutter
}

Ofelia Solís Valdespino (CUIB/UNAM)

\section{RESUMEN}

Se ex po ne el con cep to de au tor cor po ra tivo. Se afir ma que los pri me ros en ca be za mien tos cor pora ti vos halla dos en ca tálogos de bibliote cas del si glo XVII con tie nen el ger men de que una en ti dad pue da ser au to ra, res pon sable, in te lec tual de las obras que pu bli ca. Pa nizzi reconoce los encabezamientos corporativos en sus 91 reglas, pero no presenta una fundamentación de autoría corporativa. En 1850, Cres ta do ro cri ti ca a Pa nizzi y ar gu men ta por qué no pue de con si de rar se au to res a la s en ti da des. Jewett pro po ne la po si bi li dad de tra tar a las en ti da des como au to ras por ra zón de or den prác ti co, pero no les re co no ce res pon sabilidadintelectual.Pos teriormenteCutterfun damenta y enuncia la autoría corporativa.

Fi nal men te, Flet cher ob je ta a Cut ter y pro po ne re glas que en la prác ti ca con du je ronal reconocimientoimplíci to delares pon sabili dad intelectual o autoría para las entidades.

\section{SUMMARY}

The con cept of cor po ra te authorship is ex plai ned. The idea is as ser ted that the first cor po ra te en tries are found in li brary ca ta logs of the XVIIth cen tury, which con tain the germ of the re $\operatorname{cog}$ ni tion of the fact that an or ga ni za tion can be an author with in te llec tual res pon sa bility for the works it publishes. Panizzi recognizes corporate entries in his 91 rules but he does not present the basis for corporate authorship. In 1850, Cres ta do ro cri ti ci zes Pa nizzi and ar gues that en ti ties can not be con si de red authors. Jewett proposes the pos sibility of treating bodies as authors for practical reasons, but he does not give them intellectual credit. Subsecuently Cutter establishes and enunciates corporate authorship.

Finally Flet cher brings for ward ob jec tions to Cut ter but pro po ses ru les that in prac ti ce led to the im pli cit re cog ni tion of the in te llec tual responsability or authorship by organizations.

\section{EL CONCEPTO DE AUTORIA CORPORATIVA DE PA- NIZZI A CUTTER.}

¿Quésig nifica au tor cor po ra ti vo? Con el fin de ave riguar lo te nemos que re mon tar nos al pa sa do en bus ca del ori gen de este ente.

Se tie nen no ti cias fi de dig nas de que las bi blio te cas des de la an tigüe dad has ta nues tros días han con ta do con ca tá lo gos de sus colec cio nes cu yos so por tes es crip to rios han va ria do se gún la época y el lu gar, lo mis mo que la in for ma ción y el or den de ésta den tro de los registros. Se cuenta, asimismo, con información acerca del cau dal de com pi la cio nes bi blio gráficas eru di tas de bi das a biblió fi los, al gu nas tan an ti guas comola exis ten cia mis ma de pa pi ros, códices y manuscritos.

A me dia dos del si glo XVI na cen las fe rias de li bros en Ale ma nia; la de Leipzig conserva aún su bien ganadoprestigio; en ella, seña la Dahl(1) que en 1564 em pe za ron a im pri mir se ca tálogos de los libros en ven ta, ela bo ra dos por los co mer cian tes que asis tían a ellas.
Du ran te los si glos XVI y XVII se ela bo ra ron ca tá lo gos de las colecciones delasbibliotecas con ventuales, manuscritos primeroe impresos después en forma de libro en ambos casos.

Hasta este mo men to no hay no ti cias res pec to de que en es tos catálogos y bibliografías se incluyeran encabezamientos que pudieran ser considerados como antecedentes de los encabezamien tos cor porativos, talcomolos conocemos hoy día.

Eva Vero na afirma que los en ca be za mien tos cor pora ti vos apa recen en los catálogos de bibliotecas a principios del siglo XVII, (2) y en su "A his to ri cal approach to cor po ra te en tries" hace notar que en los ca tá lo gos an te rio res a las 91 Re glas del Mu seo Britá ni co hay sólo una vaga per cep ción de que los en ca be za mien tos corporativos representan autoría. Frecuentemente esos códigos es ti pu la ban un tipo de pa la bra cla ve como en tra da para las obras anó ni mas. A veces ta les pa la bras cla ve es ta ban com pues tas de la for ma in ver sa del nom bre de la ciu dad en la cual se lo ca li za ba el organismo en cuestión. En otras ocasiones se destacaba la parte del nombre que indicaba el asun to o ma te ria con cer nien te al organismo. (3) 
Aun que, Ve ro na no lo ex pli ci ta ra, los ejem plos que pro por ciona in di can que el nom bre de los or ga nis mos cor po ra ti vos se en contraban generalmente en caso genitivo en los títulos de dichos ejemplos; M. Carpenter sugiere que esta condición de genitivos pudiera con si de rar se como el fun da men to de la per cep ción de la au toría cor pora tiva. Es difícil hablar de au to ría cor pora ti vacuando los ca tá lo gos han sido cons trui dos so bre la exis ten cia del concepto de autor personal completamente desarrollado, en los cuales la existencia de encabezamientos de forma parecen estar en fran ca con tra dic ción con los en ca be za mien tos de au to res perso na les; si tua ción que se man tie ne en los mo der nos ca tá lo gos de bibliotecas y en compilaciones bibliográficas actuales. (4)

Carpenter (5), ano ta que el pri mer có di go en cu yas re glas se reconoce explícitamente la posibilidad de autoría corporativa es "Ru les for the com pi la tion of the ca ta lo gue" in clui do en Catalogue of printed books in the British Museum, vol. 1 (Londres, 1841), p. v-ix; que también es conocido como The British $\mathrm{Mu}$ seum code of ninety-one rules, adoptado por los Patronos en 1839, el cual fue compuesto en su mayor parte por Antonio Panizzi (1797-1879) con base en las 16 re glas for mu la das por Henri Harvey Baber (1775-1869), predecesor de Panizzi como con serva dor de la Bi blio te ca del Mu seo Bri tá ni co; las 16 Re glas fueron presentadas a los Patronos del Museo el 26 de abril de 1834, no contenían disposiciones para corporativos y no fueron pu bli ca das; en 1838 los Pa tro nos ur gie ron a Pa nizzi para que las firmara, acción que hizo no sin antes desarrollar un código más formal.

Pa nizzi usa la pa la bra autor sólo cuan do hace re fe ren cia a per sonas, no a en ti da des, sin em bar go es ti pu la re glas para las obras de un nú me ro de ti pos de or ga nis mos cor po ra ti vos del que se ex cluyen las aca de mias, uni versidades, socie da des científicas yór denes religiosas indicando que sean asentadas bajo el nombre del lugar en el que se localizan (Regla 9). Para los organismos exclui dos la en tra da debe ha cer se bajo el nom bre ge ne ral de "Acade mias" di vi di das por el nom bre del lu gar en el cual tie ne su sede el or ga nis mo (Re gla 80). Para este tipo de obras, acor de a la Regla 83: "De ben hacer se re fe ren cias cru za das del nom bre de cualquier au tor, edi tor o co la bo ra dor de cual quie ra de las obras arri ba men cio na das si apa re cen"; es una ins tan cia es pe cial de la pro visión ge ne ral de la Re gla 64 que pres cri be una re fe ren cia “de cualquier nombre que pueda ser razonablemente comprendida con igual de re cho para ser se lec cio na da como en tra da prin ci pal, a tal entrada". Una provisión final para entrada corporativa se encuentra en la Regla 34 estableciendoque "cuan do el nom bre del autor no aparece en el título o en cualquier otra parte de la obra (parte general de la Regla 33) debe observarse lo siguiente:"

Re gla 34. "Cuan do no apa re ce el nom bre de una per so na, en tonces se prefe ri ráel de cual quier asamblea, or ga nis mo cor po ra tivo, so cie dad, jun ta, con se jo, par ti do, sec ta o de no mi na ción que aparezca en el título, sujeto a la disposición de la Regla IX, y si tal nombre no aparece (sic.) entonces se adoptará como encabezamiento de cualquier país, provincia, ciudad, pueblo o lugar que aparezca".

También se establecieron reglas para la forma de las entradas para leyes, decisiones de las cortes y similares.
Da das es tas con si de ra cio nes, pa re ce que en las re glas de Pa nizzi hay un reconocimiento a los encabezamientos corporativos, no hay todavía una racionalizaciónde esto en los fundamentos. La evidencia presente en la Regla 34 parece indicar que la entrada cor pora ti va a ve ces toma el lu gar de la en tra da del títu lo con el fin de mejorar la recuperación.

La publicación del primero y único volumen del catálogo del Museo Británico en 1841, provocó una protesta pública que finalmente fue llevada a la averiguación de una comisión parlamentaria cuyas actas fueron publicadas en 1850; las audiencias se lle va ron a cabo del 10 de ju lio de 1847 al 26 de ju nio de 1849 . Aunque se discutieron muchos asuntos ad minis trativos del Mur seo, el grue so de los tes ti mo nios tuvo re la ción con el nue vo ca tálogo. Las partes implicadas con la parte corporativa estaban centradas principalmente alrededor de las disposiciones de la Regla 8 que indica la entrada de publicaciones de sociedades bajo el en ca be za mien to ge ne ral "Aca de mias". Esto es asun to de es ti lo de en ca be za mien tos y no es re le van te aquí. La lec tu ra cuidadosa del total de volúmenes no revela discusiónalguna sobre los méritos de la entrada corporativa, hay otras discusiones que cuestionan la entrada bajo lugares o bajo "Academias". O sea que es vir tual men te uná ni me el uso de la pa la bra "au tor" para denotar personas, esto es un hecho que confirma la interpretación de las reglas aquí presentadas.

Entretanto, hubo usuarios de la biblioteca que desearon que el catálogo se siguiera publicando, pero no fue así.

AndreaCrestadoro(1808-1879), publicóanónimamente un panfle to en 1850 , don de su ge ría una es pe cie de ca tálogo or ga ni za do por número de acceso, contiene una transcripción completa del tí tu lo del ma te rial para cada edi ción en la bi blio te ca, al que se le elaboró un índice en el cual una pa la bra de sea da de la trans cripción pudiera desplegarse al inicio del índice. Este ín di ce con cebido por Crestadoro se asemeja al moderno KWOC (Key-word-out-of-context). Crestadoro criticó los encabezamientos de academias prescritos en las 91 Reglas, dice:

“El encabezamiento 'Academias', por ejemplo, no puede sin meter en confusión todas las nociones aceptadas por el momento, abarcar aquellas que en sus portadas no están designadas como 'Academias' sino como Sociedad, Asociación, Instituto, Club, Com pa ñía, Uni ver si dad y otros nom bres se me jan tes. Una entrada así no puede ser correcta, ni legitimada, menos aun observa da, a me nos que sea he cha bajo sus pro pios tér mi nos." (6)

Es laúnica referen cia de la en tra da cor pora tivaen el panfle to. Es claro que Crestadoro no piensa en los organismos corporativos como autores por que usa el ín di ce para crear todo tipo de cla ses de en tra das. De he cho, él está con ven ci do de que "mu chas per sonas no van a la bi blio te ca a bus car au to res o es cri to res, sino a estudiar materias o asuntos." Por ello los registros sugeridos por Crestadoro contienen:

1) número de acceso (progresivo dentro del catálogo)

2) títu lo y men ción de au to ría co pia da tex tual men te de la por ta da.

3) pie de imprenta

4) dimensiones del libro. (7)

Para efectos de indización, el autor y colaboradores son solamente puntos de acceso al registro. La entrada de cada registro 
no de pen de de la re la ción en tre una per so na u or ga nis mo y un libro, a me nos que esté es ta ble ci da por la trans crip ción de la por tada, acompañada por cualquier información adicional anotada porelcatalogador. Respectoalaen tradacorporativa, Crestadoro re pre sen ta el ex tre mo en en tra da de pa la bras cla ve bajo los nombres de organismos corporativos.

Char les Coffin Jewett (1816-1868), fue más allá en el re co no cimiento de la posibilidad de tratar a los organismos corporativos como au to res. No es cla ro si Pa nizzi es ta ba de acuer do con la teoría de Jewett, aunque éste declaraba que sus reglas estaban más de acuerdo con las reglas defendidas por Panizzi que con aquellas que fue ron fi nal men te san cio na das por los Pa tro nos del museo en 1839 , lo que su gie re que Jewett tuvo ac ce so al do cu men to original de las 91 Reglas. Jewett publicó On the construction of catalogues of li bra ries and their publica tions by means of se parate, stereotyped titles. With rules and examples, en Washington. En esta obra, cita parte del apéndice al reporte de los comi sio na dos. No exis te co rres pon den cia en tre Pa nizzi y Jewett, pero éste pasó unos me ses en Lon dres, don de co no ció a Pa nizzi. (8)

En esta obra Jewett ex po ne por pri me ra vez la po si bi li dad de tratar a los organismos corporativos como autores; Carpenter cita textualmente la regla correspondiente: (9)

XXII. "Las academias, institutos, asociaciones, universidades, colegios, sociedades literarias, científicas, económicas, caritativas y religiosas; los gobiernos nacionales y municipales, las asambleas, convenciones, consejos, corporaciones y otros grupos de hombres bajo cualquier nombre y de cualquier carácter que originen publicaciones, ya sea como obras separadas, o en una se rie con ti nua, bajo un tí tu lo ge ne ral, son con si de ra das y trata das como las au to ras de to das las obras pu bli ca das por ellos, y sólo en su nom bre. El en ca be za mien to es el nom bre del or ga nismo, la palabra prin ci pal es la pri me ra que no sea un ar tículo. Se hace una referencia cruzada de cual quier sustanti vo oadje tivoa la palabra principal.

Explicación 1. Si el nombre del autor aparece a la cabeza de la portada de una obra que tiene portada y paginación distintivas, publicada por tal organismo, entonces la obra se asienta bajo la Re gla XXI, debe re gis trar se dos ve ces, una por títu lo ge ne ral según la Regla XXI y otra por el nombre del autor, refiriendo, si está pu bli ca da en una se rie, al vo lu men de la se rie en el cual está contenida.

Ex plica ción 2. Los ca tálo gos de biblio te cas pú blicas de ben asentar se bajo el nom bre del es ta ble ci mien to, y si el nom bre del compilador aparece a la cabeza de la portada, debe hacerse una referencia cruzada con la entrada principal.

Explicación 3. Cuando los comités o dependencias de un organis mo pu bli can obras, el asien to se hace bajo el nom bre de la entidad principal, no bajo el de la dependencia subordinada. En ton ces, bajo Estados Unidos secolocan to dos los docu mentos pú bli cos que se pu bli can a ex pen sas de los Es ta dos Uni dos, ta les como documen tos públi cos re gu lares, o de de partamen tos, oficinas o co mi tés. Es tos títu los, cuan dolle gan a ser nu me ro sos pueden clasificarse y arreglarse convenientemente en el catálogo.
Bajo el mismo principio, las publicaciones de sociedades literarias o re li gio sas re la cio na das con co le gios y uni ver si da des secatalogan bajo el nombre de los colegios, etc., con referencias cruzadas de los nombres de las sociedades.

Ex pli ca ción 4. Bajo esta re gla, las li tur gias, li bros de ora cio nes, breviarios, misales, etc., se colocan bajo el nombre en in glés de la comunión, orden religiosa o denominación, bajo cuya autoridad son pre para dos y publica dos. Las obras si mi la res de au to res individuales, se asientan bajo sus nombres." (10)

Por lo tanto, podemos afirmar que Jewett no considera que los organismos corporativossean autores, sino que pue den ser consi de ra dos como ta les y tra ta dos como si lo fue ran para efec tos de catalogación. Cuando habla de los catálogos de bibliotecas está im pli can do que la for ma de la pu bli ca ción pue da ser un ele men to importante, además de la autoría para determinar el encabezamiento elegido en el asiento principal.

La razón del tratamiento de obras publicadas por los gobiernos no es cla ra dado que Jewett fue por lar go tiem po con ser va dor de documentos gubernamentales de la colección Smithsonian, así que te nía idea de la can ti dad de asien tos que en tra rían bajo Estados Unidos, sin subdivisiones. Dice que hay que clasificarlos bajo el en ca be za mien to deEstados Unidos, pero no in di ca cómo hacerlo. Si afirma que Estados Unidos puede ser tratado como autor de los documentos gubernamentales porque estos documentos son producidos a expensas del gobierno de los Estados Uni dos, es una in fe ren cia in co rrec ta. Jewett in sis te en asen tar las publicaciones de las sociedades de colegios bajo el nombre del colegio; tales sociedades, en tanto estén formadas por personas re la cio na das con el co le gio pue den no es tar sub ven cio na das por es tos co le gios, o even tual men te, no man te ner bue nas re la ciones con la ad mi nis tra ción de los mis mos, de tal suer te que cae en una tram pa y no lle ga a mos trar en es tos en ca be za mien tos quién es en realidad el organismo responsable de la publicación.

Respecto a las obras litúrgicas, es necesario recordar que éstas son anteriores al origen de las diversas órdenes religiosas. Esto hace pen sar que Jewett no tuvo cla ri dad res pec to al ori gen de las obras litúrgicas, o bien, que la re gla no tie ne una re dac ción adecuada. La corrección sería: "La forma inglesa del nombre de la comunión, orden religiosa o denominación bajo cuya autoridad han sido adop ta das y pu bli ca das." Esta ha sido la raíz de mu chos problemas concernientes al tratamiento de las obras litúrgicas.

En 1876, Charles Ammi Cut ter pu bli có la pri me ra edi ción de su Rulesforaprinted dic tionaryca talog (Wa shing ton, 1876), y cuyas revisiones, propiamente nuevas ediciones se publicaron en 1889,1891 y 1904 . Este có di go es con si de ra do como el pi lar sobre el que se construyó la tradición catalográfica angloamericana. En la se gun da edi ción en con tra mos de la pá gi na 27 a la 31, las re glas con cer nien tes a los or ga nis mos cor pora ti vos; és tas van de la Re gla 39 (prin ci pio ge ne ral a la re gla 56 (pu bli ca cio nes de sociedades)).

\section{CORPORATIVO}

\section{Principio General}

39. Los gru pos de hom bres son con si de ra dos como au to res de las obras publicadas en su nombre o bajo su autoridad. 
La prin ci pal di fi cul tad con res pec to a los gru pos de hom bres está en de ter mi nar 1) cuál es su nom bre y 2) si el nom bre u otra pa labra seráel en ca be za mien to. Res pec to a 2), los ca tálo gos publicados hasta la fecha pueden ser considerados como una serie de ex perimen tos. Aún no se ha es table ci do un uso sa tis fac to rio. Los nom bres de lu ga res siem pre tie nen mu cha de man da para ser usados como encabezamientos, pero para asentar las publicaciones de to dos los gru pos de hom bres bajo los nom bres de los lu ga res con los que están relacionados es impulsar una práctica conveniente que hasta ahora ha devenido en inconvenientes y ha permitido muchas reglas fuera de la armonía del catálogo." (11)

En este tex to hay un pe que ño fun da men to para tra tar a las en ti dades corporativas como autores. Cutter estableció en su definición de autor lo siguiente:

"Autor, en el sentido más restringido, es la persona que escribe un li bro; en el sen ti do más am plio, pue de ser apli ca do a la per sona responsable de la existencia de un libro por reunir en uno de los es cri tos de varios au to res (usual men te lla ma doel edi tor, más propiamentedeberíalla mársele elcolector). Los gru pos de hombres (sociedades, ciudades, cuerpos legislativos, países) son con si de ra dos los au to res de sus me morias, ac tas, re vis tas, de bates, reportes, etc.” (12)

En la cuarta edición se encuentra, en la introducción al capítulo para cor po ra ti vos, una fun da men ta ción de los con cep tos de Cutter; es por ello que se con si de ra im por tan te in cluir al gu nos párrafos. (13)

"Un ar tícu lo en Library Journal $(21: 493,494)$ se oponía al principio de autoría corporativa como si se tratara de una superstición bibliotecaria, y recomendaba la práctica alemana, que considera 'todas las obras publicadas pororganis mos cor porati vos como anó ni mas, para pro pó si tos de en tra da', cuan do no tienen un autor individual."

De se guir se el cri te rio ale mán, las obras pro duc to de cor po ra ciones quedarían dispersas en el catálogo dado que se asentarían bajo la palabra que apareciera primero en el título.

Dehe cho, la prác tica ame ri ca na tie ne dos ven tajas. La pri me ra, con si de ra que es tos or ga nis mos son au to res de sus ac tas, etc., y de sus co lec cio nes vis tas és tas como un todo; la se gun da por que es más conveniente para el servicio al público que todas las obras pro du ci das por una so cie dad o por un go bier no es tén re ut nidas, esta ventaja es más evidente aun en los catálogos tipo diccionario.

Cutter hacíareferencia a la lista Kayses en la que, para localizar las publicaciones de so cie da descien tíficas ha bía que bus carbajo 41 palabras diferentes; esto requiere de gran paciencia, acuosidad y es prác tica men teim posible pro porcionarservicios eficientes en estas condiciones de operación.

Asi mis mo, para Cut ter hay dos cla ses de pu bli ca ciones gu ber namen ta les: aquéllas que son bien iden ti fi ca das por un au tor per sonal, y las anónimas. Las primeras no representan problema: "Pero, las Reglas in di can que si fue ran pu bli ca das por el go bierno, deben apa re cer tam bién ya sea en for ma com ple ta o por una referencia, acorde a las circunstancias, bajo la dependencia guberna men tal que las publica. Una biblio te ca pe que ña bien pue de omi tir esto... pero una bi blio te ca gran de no estábien ca ta lo ga da a me nos que ten ga lis ta dos de re fe ren cia de to das las obras rea li zadas bajo la responsabilidad de cada dependencia gubernamental". Como se aprecia, para él era relevante el control de las publica ciones gu ber na men ta les, de ahíla ne ce si dad de hacerentradassecundarias para este tipo de corporativos cuan do son los patrocinadores de las publicaciones.

En cuanto a las publicaciones gubernamentales anónimas, este autor distingue tres clases: 1) "Son diarios de debates, reportes, etc., de cuer pos le gis la ti vos, de los cua les mi obje tar igual men te ad mi te que el go bier no es el au tor y los co lo ca bajo el país (a di feren cia de los ale ma nes); o 2) se re la cio nan al país, en cuyo caso el objetar los coloca también bajo el país, pero en una división de materia y no bajo el de par ta men to;o3) noes tán re la cio na das con el país. En cuan to a 1) es ta mos de acuer do; res pec to a 2) no ten go obje ción de nin gu na cla se para asen tar las com ple ta men te bajoel en ca be za mien to de país- ma te ria único, prove yen do a esta en trada de una re fe ren cia bajo el nom bre del de parta men to... 3) po cos trabajosanónimos publicados por un departamentoy que no están re la cio na dos con el país... de ben asen tar se bajo la pri me ra pa labra... me parece que debería haber un asiento bajo el de par ta men to que más aun en el caso de obras pu bli ca das con sus nombres de autor, debe suponerse que adoptan las opiniones de la obra y asumen su responsabilidad."

Para la elaboración de las Reglas, Cut ter trabajó en loca li zar los principios simplesalrededor de las cuales fue raposibleagrupar las prácticas catalográficas más convenientes. "Uno de esos prin ci pios es la au toría cor po ra ti va y la res pon sabilidad edi torial. No tengo nada aún que me convenza de que esto no es bueno, dado que corresponde de hecho, al que muchas sociedades son au to ras de sus ac tos y co lec to ras de sus se ries; esto es con ve niente en la prác ti ca para una ca ta lo ga ción com ple ta; y para una ca talogación incompleta se admiten las economías que producen to dos los efec tos que al ob je tar re cha za en este prin ci pio con ninguna de las desventajas de su método."

Como puede verse, los alemanes establecieron el asiento bajo el pri mer sus tan ti vo del tí tu lo para las obras anó ni mas. El oponen te de Cut ter, Flet cher, es ta ble ció, en rea li dad, la en tra da del tí tu lo pero bajo el nom bre de la so cie dad: Ro yal So ciety, proceeding of y no Proceedings of the Royal Society, como sería la regla alemana.

Más ade lan te, a pro pó si to de los cam bios de nom bre de una misma institución merced a influencias de idiomas extranjeros, reflexiona: “... desde luego, uno puede acabar parcialmente con esa ob je ta ble dis per sión de obras que de ben ser asen ta das jun tas po nien do to das las so cie da des pri me ro bajo el nom bre del lu gar donde tienen su sede; pero en aquellas ciudades que tienen muchas sociedades habrá cierta cantidad de conflictos en cuanto a for mas di fe ren tes y se pa ra ción de obras de aquellos au to res cuyo nom bre ha apa re ci do en idio mas di fe ren tes o en for mas dis tin tas en las portadas, y si esto es evitadomediante la adop ción de una for ma úni ca para to dos, ¿qué es esto sino el aban do no de la idea de entrada de título y el regreso a la entrada de autor?"

Así es, en la prác ti ca se dan una se rie de ca sos con cre tos, las propuestas de Fletcher llevan finalmente al reforzamiento de los 
con cep tos de Char les Ammi Cut ter acer ca de las ven ta jas de trabajar, catalográficamente, alrededor de la autoría corporativa.

Po de mos re ca pi tu lar: en el si glo XVII apa re cen los pri me ros encabe za mien tos cor pora ti vos aun que en los ca tálogos de biblio tecas que datan de esas fechas solamente se percibe vagamente la idea de que ta les en ca be za mien tos pue den re pre sen tar res pon sabilidad intelectual o autoría.

Es Antonio Panizzi, en 1839, quien reconoce explícitamente la autoría corporativa en las 91 Reglas del Museo Británico en las que usa el tér mi no au tor para re fe rir se a per so nas, y sin em bar go establece claramente reglas para la entrada de las publicaciones de cierto tipo de corporaciones. Hablamos aquí de las reglas 9, $33,34,80$ y 83. Pa nizzi re co noce los en ca be za mien tos cor porati vos, pero no hay aún una ra cio na li za ción que fun da men te di chos encabezamientos.

\section{NOTAS BIBLIOGRAFICAS}

1. DHAL, Svend. Historia del libro / Tr. del danés por Alberto Adell; adiciones es pa ño las de Fer nan do Huar te Mor ton. — Madrid : Alianza Editorial, c1982. - (Alianza Universidad ; 178) p. 141.

2. VERONA, Eva. Corporate headings : their use in library catalogues and national bibliographies : a critical study. - London : IFLA , Committee on Cataloguing, 1975. p. 8.

3. —_- "A historical approach to corporate entries." — En Libri. — v. 7 (1956) p. 27

4. CAR PEN TER, Mi chael.Corporate autorship: its role in librarycataloging. - Westport, Conn. : Gre enwood, c1981. - (Con tri butions in librarianship and information science ; no. 34) p. 7-8.

5. Ibid. p. 100

6. Ibid. p. 10

7. Loc. cit.

8. CARPENTE. Op. cit. p. 101.

9. Ibid. p-10-11

10. Ibid p. 11

11. CUT TER, Char les Ammi.Rulesforadictionarycatalog. — 2nd. ed. - Wa shing ton, D.C., : Go bernment Prin ting offi ce, 1889. p. 27

12. Ibid. p. 9

13. CUT TER, Char les Ammi.Rulesforadictionary catalog. - 4th. ed. with co rrec tions and addi tions. - Wa shing ton, D.C. : Bu reau of Education, 1904. - p. 39-41
Andrea Cres ta do ro en 1850 pu bli ca un pan fle to en el que cri ti ca los encabezamientos de las academias prescritas por Panizzi. Para Cres ta do ro, crea do ra de los mo der nos KWOC, los cor porativos no son autores.

Charles Coffin Jewett publicó más tarde On the construction of catalogues... en la que ex po ne la po si bi li dad de dar a los cor po rativos tratamiento de autores, particularmente en la regla XXII.

Charles Ammi Cutter, en 1876, publicó las célebres Rules for a printed dictionary catalog, obra fun da men tal en la tra di ción cacorporativa.

Flet cher, años des pués, ob je tó el con cep to de au toría cor po ra ti va y pro pu so re glas más acor des con la tra di ción pru sia na pero que, ya en la práctica llevaron, como la señaló Cutter, al establecimien to de for mas úni cas para cada en ti dad, y al re co no ci mien to implícito del concepto de autoría corporativa. 